\title{
A review on synchrophasor technology for power system monitoring
}

\begin{abstract}
The Phasor Measurement Unit (PMU) is the heart of smart grid system as it provides the data such as voltage and phase angle measurements of all buses of the system and thereby maintaining the system observability. In this context, this paper summarizes the various research based on PMU for complete observability and monitoring of integrated power system. The survey indicates that most of the recent researches are focusing on optimal PMU placement (OPP) rather than design and modeling of PMU considering various cases. Moreover, the state estimation using synchrophasor technology are also presented as addition objective to obtain the optimal number of PMU that need to be installed in the system for power system analysis and economic benefits of the system. The trend of research based on synchrophasor technology are evolving for real-time power system monitoring application where it also covers for dynamic power system assessment.
\end{abstract}

Keyword: Phasor measurement unit; Synchrophasor measurement technology; Optimal PMU placement; State estimation; System observability 